\title{
Avaliação da Qualidade dos Serviços de Saúde: Notas Bibl iográficas
}

\author{
Eduardo J.F. B. dos Reis* \\ Fausto $P$. dos Santos* \\ Francisco Eduardo de Campos ** \\ Frañcisco de Assis Acúrcio * \\ Marcelo T. T. Leite* \\ Maria Léa $C$. Leite* \\ $M$ ariangela $L$. Cherchiglia * \\ Max A. dos Santos*
}

No momento em que se discute a reorganização dos serviços de saúde no Brasil, a avaliaçāo da qualidade destes serviços ganha importância. Nesta revisâo, buscamos os antecedentes históricos da avaliação dos serviços de saude e apresentamos as propostas contemporâneas de sistematização da avaliação de qualidade. Através da análise dos trabalhos revisados, identificamos duas grandes linhas metodológicas de acordo com seu objeto de estudo: Estudos Corporativos Racionalizadores e Estudos Antropossociais. A maioria destes se referenciam nos trabalhos desenvolvidos por Avedis Donabedian, autor que, nos últimos vinte anos, vem desenvolvendo estudos de avaliaçāo dos serviços de saúde.

* Pesquisadores do Nescon, Es. pecialistas em Medicina Social

\section{** Professor Adjunto da Facul.} dade de Medicina da UFMG

*** Esta revisão constitui parte do trabalho desenvolvido na pesquisa "Produ tividade e Resohutividade dos Serviços de Saúde Frente a Política de Recursos Humanos" do Programa de Estudo e Pesquisa de Política de Saúde (PEPPS) Minister io da Saúde-convênio UFMG/Nescon/ MS de junho 187, e da tese de doutoramento de Francisco Eduardo de Campos, "Resohutividade: Uma Aproximaçăo à Avaliação Qualitativa". Ensp/ Fiocruz, maio de 1988.

\section{INTRODUÇÃO}

Neste momento, em que se discute a reorganização dos serviços de saúde do Brasil (AIS, VIII Conferência Nacional de Saúde, CNRS, SUDS), o tema da qualidade dos serviços, em seus termos mais amplos, ganha relevância.

$O$ objetivo deste trabalho é apresentar uma revisão bibliográfica, no sentido de subsidiar as discussōes em andamento referentes à qualidade dos serviços de saúde no Brasil ***, até então pouco estudada na literatura nacional. Apesar desta discussão estar presente desde a antiguidade, desenvolveu-se com mais vigor neste século e em especial, nas últimas décadas, nos países capitalistas centrais.

\section{ANTECEDENTES HISTÓRICOS}

A análise da literatura sobre a avaliação de serviços de saúde demonstra que sempre existiram mecanismos de avaliaçāo da qualidade da prática médica e dos serviços de saúde, caracterizados pela formação

Cadernos de Saúde Pública, RJ, 6(1): 50-61, jan/mar, 1990 
tanto da opinião pública quanto dos conselhos corporativos. Estes mecanismos são tão antigos quanto esses serviços (16).

O estudo realizado pela OPS acerca de "Evaluación de la Eficacia y la Seguridad de las Tecnologías Médicas", relata que "o uso da estatística e da avaliação científica dos cuidados médicos se desenvolveu lentamente, pois, em geral, não era considerado aspecto essencial da Medicina"' (23).

Talvez a primeira sistematização contemporânea* de um mecanismo de avaliação do ensino - e, por conseqüência, da prática : :dica - tenha sido o conhecido relatório Flexner (13), publicado em 1910, sob o patrocínio da Fundação Carneggie. Neste relatório, Flexner atenta para a necessidade de controle do exercício profissional, ao avaliar a educação médica e denunciar, de maneira muito enfática, as precárias condiçōes da prática profissional.

$\mathrm{Na}$ trilha aberta pelo relatório Flexner e respondendo, fundamentalmente, às mesmas determinações, surge ou tro trabalho clássico sobre avaliação dos servi* ços de saúde. Trata-se do trabalho de Codman "A Study in Hospital Efficiency: the first five years" (25). Publicado em 1916, este trabalho apresenta proposta de metodologia de avaliaçāo rotineira do estado de saúde dos pacientes, para estabelecer os resultados finais das intervenções médicas intra-hospitalares. Relatava, caso a caso, a que se poderia atribuir o sucesso ou $o$ insucesso do tratamento, qual momento em que se poderia ter uma avaliação definitiva do caso e, por fim, o que poderia ocorrer até mesmo um ano depois de terem sido realizadas as intervençōes. Sob $a$ influência do trabalho de Codman, o Colégio Americano de Cirurgiōes assumilı a responsabilidade pela avaliação da qualidade das práticas cirúrgicas e doi hospitais. Realizou, então, um famoso estudo, no qual registra que, entre os aproximadamente 800 hospitais examinados com mais de 100 leitos, menos de 135 tinham algum grau razoável de qualidade. Como conseqüência deste trabalho e dos estudos desenvolvidos por Codman, sria-se, em 1928, o Hospital Standartization Program, que é o embrião da Joint Comission of Accreditation of Hospitals (JCAH) (25).

Com os crescentes custos da atenção médica, em especial nos países centrais, e o aumento da complexidade da atenção (uso indiscriminado de tecnologia e procedimentos médicos cada vez mais sofisticados), acontece um impulso objetivo para a expansão de trabalhos e pesquisas de avaliação da qualidade e dos custos da atenção médica.

$\mathrm{Na}$ Inglaterra, neste século, o atendimento gratuito do National Health Service (NHS) leva a uma crescente demanda por serviços de saúde, o que inclui Cadernos de Saúde Pública, RJ, 6(1): 50-61, jan/mar, 1990
* Para revisão das práticas de avaliação em saúde, em perfodos mais remotos, ver: 1) Rodes, Philip. An outline history of Medicine. Butter worths, 1985. Kent, England. 2) Focau $1, \mathrm{Mi}-$ chael. Microfísica do Poder. Rio de Janeiro, 1979, Graal. 
consultas médicas, exames subsidiários e medicação. Dada a limitação de recursos utilizados para o custeio do NHS, buscou-se otimizar sua aplicação, surgindo preocupaçōes com estudos da eficácia e eficiência dos procedimentos diagnósticos e terapêuticos (17).

Nos EUA, a partir de 1960 , com a implantação de programas sociais federais de atenção à saúde (Medicare e Medicaid), o reembolso dos custos exigiu uma avaliação do cuidad o médico, $t$ anto ao nível hospitalar como para credenciamento de unidades ambulatoriais (PSRO-Organização,Estandartização e Revisão Profissional) $(12,14)$. Para essa avaliaçăo, utilizou-se de conhecimento acumulado nos comitês de peritagens e experts (Peer Review) (7).

Segundo Perez Arias (24), "o incremento desenfreado dos custos ultrapassou o previsível e desejável, ainda que em países ricos. $O$ gasto anárquico beneficia alguns setores e prejudica outros, sem que isto signifique maior eficácia. O aumento global dos custos e a incorporação de grande quantidade de tecnologia para o diagnóstico e tratamento de enfermidades não têm dado, como resultado, a redução da morbimortalidade. A qualidade da atenção médica sofre uma evidente deterioração devido, entre outras causas, à diluição da responsabilidade que é transpassada e atomizada por numerosos atores intervenientes". Estas são razões suficientes para se buscar o controle dos custos e da qualidade a atenção médica, principalmente tendo como pano de fundo a crise econômica mundial.

A tendência ao aumento dos custos do setor saúde é, na verdade, universal. Vários autores trabalharam este problema, bem como analisaram suas causas, ligadas ao fato de que o cuidado médico, além de difícil padronização, não possui valor de troca, mas tão-somente valor de uso para aquele que o consome. Alia-se a este fato que, ao contrário do que ocorre na evolução da manufatura para a indústria, a incorporação tecnológica não substitui o trabalho vivo consumido no cuidado, nem tecnologias mais antigas. A este propósito devem ser consultados os trabalhos de Donnangelo (10), Arouca (3) e Nogueira (22).

Nas últimas duas décadas, os estudos sobre a qualidade dos serviços de saúde têm-se baseado em uma ou mais categorias propostas por Donabedian (8). Utilizando as experiências de avaliação desenvolvidas por Flexner (13) e Codman (25) e tendo como referencial a teoria dos sistemas, este autor sistematizou a a valiação qualitativa de atenção médica em três aspectos: estrutura, processo e resultado.

Existem várias propostas de agregação dos estudos de avaliação dos serviços de saúde, Por exemplo: Sonis e Paganini (27)propõem que os métodos de avaliaçẫo podem se dar através de avaliações administra- 
tivas, sem estudo especial, ou através de investigações avaliativas. Nas duas abordagens, as possibilidades de estudo são ainda aquelas apontadas anteriormente, ou seja: estrutura, processo e resultado. Enquanto os estudos de estrutura se desenvolvem, fundamentalmente, nos níveis institucionais e dos sistemas de atenção à saúde, os estudos de processo e resultado podem ter como referência o indivíduo, grupos de usuários ou toda a população.

\section{BASES TEÓRICAS DOS ESTUDOS ATUAIS}

O autor que mais se aproxima de uma proposta de avaliação da qualidade dos serviços de saúde é A vedis Donabedian (8), que vem publicando uma série de importantes trabalhos para a literatura médica nos últimos 20 anos. Dificilmente sāo encontrados artigos na literatura que não se baseiem ou citem, com destaque, o trabalho desse autor. A avaliação qualitativa do cuidado médico poderia se dar em três de seus componentes: estrutura, processo e resultado. Podem existir combinaçōes entre esses três componentes, como se verá oportunamente. Como seria de se esperar, entretanto, as metodologias propostas estão muito vinculadas à modalidade de organização da prática médicà norte-americana, estando sempre presente a perspectiva de melhoria qualitativa dos padröes, visando a um "autogoverno" das profissōes.

Em artigo publicado em 1978, Donabedian (9) diz que a avaliaçâo dos serviços comporta sempre duas dimensões: 1) desempenho técnico, ou seja, a aplicação do conhecimento e da tecnologia médica de modo a maximizar os benefícios e minimizar os riscos, de acordo com as preferências de cada paciente; 2) 0 relacionamento pessoal com o paciente, de modo a satisfazer os preceitos éticos, as normas sociais e as legítimas expectativas e necessidades dos pacientes. A partir da interação entre o cliente e o médico, existe um processo complexo, que vai desde componentes comportamentais até componentes técnicos muito específicos.

Segundo Donabedian, "o objetivo da avaliação da qualidade é determinar o grau de sucesso das profissões relacionadas com a saúde, em se autogovernarem, de modo a impedir a exploração ou a incompetência, e o objetivo da monitorização da qualidade é exercer vigilância contínua, de tal forma que desvios dos padrōes possam ser precocemente detectados e corrigidos" (9).

O estudo da estrutura avalia, fundamentalmente, as características dos recursos que se empregam na atenção médica e considera os seguintes componentes: medidas que se referem à organização administrativa 
da atenção médica; descrição das características das instalaçōes, da equipe médica disponível, fundamentalmente em relação à sua adequação com as normas vigentes; perfil dos profissionais empregados, seu tipo, preparação e experiência. Os estudos que se baseiam somente na estrutura têm sido pouco considerados por este autor (9).

A avaliação de processo descreve as atividades do serviço de atenção médica. Esse tipo de avaliaçāo está orientado, principalmente, para a análise da competência médica no tratamento dos problemas de saúde, isto é, o que é feito para o paciente com respeito à sua doença ou complicação particular. A avaliação do processo compara os procedimentos empregados com os estabelecimentos como normas pelos próprios profissionais de saúde. Geralmente os critérios são estabelecidos pelo estudo da eficácia de práticas médicas rotineiras. Segundo Donabedian (9), a metodologia dos estudos de processo pode ser dividida de duas maneiras: observação direta da prática e os estudos baseados nos registros médicos.

A avaliação do resultado descreve o estado de saúde do indivíduo ou da população como resultado da interação ou não com os serviços de saúde. Como, em termos de saúde, os resul tados se devem a muitos fatores, a sua medida e avaliação constituem o que existe de mais próximo em termos de avaliação do cuidado total (9). Considerando-se a natureza multifacetada do estado de saúde, existem muitas metodologias que utilizam medidas de resultado seguras e validas. Entre elas, incluem-se medidas de capacidade física e estado funcional, inventários de saúde mental, medida do impacto das doenças sobre o comportamento dos indivíduos (Perfil de Impacto da Doença (SIP)) e medida de percepção pessoal da saúde geral.

Brook (4) e colaboradores vêm trabalhando no desenvolvimento de uma metodologia que estude a relação entre processo e resultado como indicador da qualidade do cuidado, no entendimento de que os serviços prestados ao paciente guardam alguma relação com seu subseqüente estado de saúde. A forma de se alcançar este objetivo seria, segundo Donabedian (9), os estudos de trajetórias e traçadores ("tracers"), que se baseiam na seleção de uma (ou mais) doença ou condição, podendo-se entāo fazer o acompanhamento do paciente desde a sua chegada ao serviço de saúde até o presumível cuidado final.

As investigações que se referenciam nas categorias de Donabedian, atualmente, têm proposto um modelo in tegrativo, em que se avaliam as relações entre estado de saúde, qualidade do cuidado e gastos de recursos. Brook (4) sugere que: “... um grande esforço de pesquisa (talvez o mais importante deles) seria a 
tentativa de integrar: a) a eficácia do cuidado onde as circunstâncias são mais favoráveis à produção de bons resultados; b) a efetividade do cuidado no curso diário dos eventos; c) as variaçōes das características populacionais no uso do serviço e as variaçōes intimamente relacionadas à qualidade do cuidado, medidas em termos de resultados no paciente; d) e, finalmente, os níveis de qualidade de cuidado nos termos mais amplos possíveis".

Pela literatura podemos notar que os diferentes estudos de avaliação dos serviços de saúde hoje desenvolvidos referenciam-se em uma ou mais categorias donabedianas. Por isso os reunimos em dois grandes grupos: a) Estudos Corporativos Racionalizadores; b) Estudos Antropossociais.

\section{A) Estudos Corporativos Racionalizadores}

Abrange a auditoria médica, análises de custc, avaliação de eficácia e segurança de tecnologia médica.

\section{Auditoria Médica}

A auditoria médica tem sua origem no próprio exercício da prática médica liberal. Mas é com o trabalho de Flexner de 1910 e o de Codman em 1916 que se tem, talvez, a primeira tentativa de sistematização de um mecanismo de avaliação dos serviços de saúde $(13,25,21)$.

É sabido que a expansão da auditoria médica se produziu basicamente com o crescimento dos seguros médicos e da seguridade social, o que levava a uma necessidade de avaliar os serviços prestados e também racionalizar os custos. Alia-se a este fato o objetivo subjacente de corporações que procuravam obter resguardo profissional. Como assinala Busso, em "Perspectivas de 1". Auditoria Médica" (5), a auditoria foi aceita pelos médicos porque significava levantar um baluarte preventivo contra as açōes sociais e judiciais por "má prática".

Segundo Donabedian (7), a auditoria é necessária porque, para assegurar a qualidade do cuidado, náo é suficiente confiar em mecanismos informais de auto- avaliação. Há, segundo ele, três formas de se fazer uma auditoria contínua e periódica das atividades profissionais. São elas: a) interna ou externa; b) administrativa ou profissional; c) por revisāo de casos ou estatística. E Donabedian chega à conclusão que a abordagem mais eficiente e eficaz para a revisâo da atividade profissional envolve uma combinação da análise estatística e da revisão de casos. A análise estatística possibilita uma rápida revisão geral da situação, 
identificando áreas de possíveis debilidades que seriam, então, analisadas detalhadamente pela revisão de casos.

\section{Análise de Custos}

$\mathrm{Na}$ segunda metade da década de 60 , to1 introduzida na literatura médica a discussão entre Análise de Custo-Benefício (CBA)/Análise de Custo-Efetividade (CEA) (32). Mas é com a publicação em 1972 de "Effectiveness and Efficiency: Randam Reflections on Health Services" de Cochrane (6) que há um profundo impacto no crescimento dos estudos de CBA/ CEA voltados para a avaliação dos serviços de saúde. Entre os trabalhos podemos destacar os dois artigos publicados em 1974 por Klauman (19), com a metodologia e aplicações do CBA,e os publicados por Weinstein (30), três anos após, com a discussão da metodologia e aplicações da CEA. A partir de então há um aumento acentuado de estudos de CBA/CEA na avaliação de serviços de saúde.

Análise de Custo-Benefício, Análise de Custo-Efetividade e Análise de Custo-Utilidade (CUA) são técnicas analíticas formais para a comparaçāo das conseqüências positivas e negativas na utilização de recursos. $\mathrm{Na}$ realidade, nada mais são do que tentativas de se pesarem logicamente os prós e os contras de uma decisão (29).

$\mathrm{Na} \mathrm{CBA}$, as conseqüências positivas dos programas avaliados (os benefícios), assim como os custos, são medidos em unidades monetárias. Is to permite, conceitualmente, uma avaliação do valor inerente a um programa, assim como uma comparação entre programas semelhantes.

Já a CEA mede as conseqüências positivas não em termos monetários, mas em uma outra unidade qualquer, tal como anos de vida poupados, dias de morbidade ou mortalidade evitadas etc. Permite, portanto, a comparação de custo por unidade de efetividade entre programas com o mesmo objetivo, mas não entre programas com objetivos diferentes, porque a efetividade das medidas de resultado ("outcome measures") difere. Isto porque, apesăr de a medida final ser a mesma, os parâmetros utilizados em cada programa não são comparáveis.

A CUA enfoca, com atenção no resultado, a qualidade da saúde obtida por um programa de saúde ou tratamento. Os resultados são geralmente expressos como custo por ganho em qualidade de anos de vida ajustados (QALY) (11).

Cadernos de Saúde Pública, RJ, 6(1): 50-61, jan/mar, 1990 
Avaliação de Eficácia e Segurança de Tecnologia Médica

$O$ desenvolvimento e o uso das tecnologias médicas durante as últimas décadas têm crescido rapidamente. É inegável que inovaçōes tecnológicas têm contribuído para a queda, em todo o mundo, das taxas de mortalidade e morbidade. Entretanto, "ainda que as novas tecnologias tenham produzido mudanças no estado de saúde de muitas populações, é impoit note reconhecer que muitas dessas mudanças têm um custo muito elevado em relarão aos benefícios produzidos. Muitas dessas tecnologias introduzidas pela medicina de beneficio marginal e implicam riscos que necessitam ser considerados nas decisōes" (23).

É neste contexto que, em 1978, o Office of Technology Assessment (OTA), a pedido do Cugresso dos EUA, revê a eficácia e segurança de 17 diferentes casos de tecnologia médica. OOTA conclui que muitas des:is tecnologias não têm adequâda avaliação de eficácia e segurança antes de serem difundidas. Entı as partes estudadas, algumas podem estar sendo equivocadamente utilizadas, não se esperando mudança apreciável em um curto espaço de tempo (23).

\section{B - Extudos Antropossociais}

Esta veriente, que tem os individuos es grupamentos sociais como base de sua metodologia para a valiação dos serviços de saúdc, desenvolveurse, principalmente, a partir da década de 70 . Seus eixos principais são o estudo de acessibilidade e da satisfação dos pacientes.

\section{Acess ibilidade}

A acessibilidade, utilizada como um indicador da qualidade do serviço de saúde, tem seu marco a partir da década de 60-70, diante da contradição existente, nos EUA, entre a disposição governamental de expansão dos serviços de saúde e a não-correspondente eqüidade destes. Segundo Aday (1), em 1982, a acessibilidade da população americana aos serviços de saúde continuava a ser determinada, em última instância, pela rerida do indivíduo.

Existem duas vertentes principais na literatura que se baseiam na acessibilidade como metodologia de avaliação do acesso com as características da po pillação (renda familiar, cobertura providenciária, atitudes frente ao cuidado médico) ou com os sistemas de saúde (distribuição a oganização dos serviços, relaçöes de poder etc). A segunda vertente relaciona a 
avaliação do acesso aos indicadores de resultado da passagem do indivíduo pelo sistema (padrão de utilização e satisfaçäo). Segundo pesquisadores dessa última vertente, essa metodologia permite "validações externas" da importância do sistema e das características individuais (2).

Segundo Donabedian (2), dois aspectos da acessibilidade podem ser distinguidos: o sócio-organizacional e o geográfico. Nos aspectos sócio-organizacionais, incluem-se tanto os atributos referentes aos recursos quanto à organizaçāo, que podem facilitar ou dificultar os esforços do cliente em obter o cuidado. Nesse aspecto, podem-se incluir variáveis referentes ao profissional de saúde tais como sexo e especialização e também as preferências do paciente. A acessibilidade geográfica, por outro lado, refere-se ao "isolamento espacial", uma função do tempo e da distância física a ser percorrida pelo paciente para obter o cuidado.

Garro e Cols (15), utilizando a antropologia como referencial metodológico, chegam à enfática conclusão de que a acessibilidade ao serviço de saúde é o fator primordial à sua utilização ou não, superando mesmo barreiras étnicas e culturais.

\section{Satisfação}

Ainda que a satisfação do paciente seja englobada freqüentemente no processo ou resultado final do cuidado, segundo Lebow (20) esta rotulação é uma simplificação, porque a percepção individual do cuidado é mais complexa do que a avaliação do processo ou resultado final. Vários fatores afetam a percepção do paciente. Entre eles se destacam: as experiências anteriores de cuidado médico recebido, em que condições elas se deram e o seu estado atual de saúde. Esses fatores não são levados em consideração quando se utiliza o estudo de processo e resul tado como metodologia de avaliação dos serviços de saúde.

A partir dos resultados preliminares de vários estudos realizados, utilizando a satisfação do paciente como medida da qualidade dos cuidados de saúde $(20$, $28,18,26)$, pode-se inferir que os fatores mais importantes, na percepção dos pacientes, foram: 1) in teresse pessoal do médico pelo paciente; 2) acessibilidade; 3) "bons médicos"; 4) acompanhamento por pessoal "bem treinado"; 5) informação dos médicos; 6) pessoal "solícito"; 7) privacidade.

Donabedian (20) sugere que o estudo da satisfação do paciente é o mais importante objetivo no estudo do cuidado médico, apesar de não poder ser um indicador direto ou indireto, mas apenas aproximado, da qualidade do cuidado à saúde. $\mathrm{O}$ mesmo autor inclui 
a satisfação dos profissionais de saúde como uma importante dimensão na avaliação global da qualidade do cuidado.

\section{CONCLUSĀO}

A partir deste estudo podemos concluir pela necessidade de se aprofundar na sistematização do conhecimento nesta área, principalmente buscando pontos de con tato ou reflexōes que possam servir de subsídios ao desenvolvimento dos estudos voltados para a realidade brasileira. Nessa perspectiva destacamos os seguintes pontos:

1) Nos países centrais, o desenvolvimento da avaliação qualitativa deu-se, num primeiro momento, sob o impulso das corporaçōes médicas na busca do controle das más práticas e do resguardo profissional. A auditoria médica continua como mecanismo importante deste controle. No Brasil, por outro lado, a forma do desenvolvimento das entidades profissionais não incorporaram essa prática.

2) A existência de seguro privado e/ou controle da sociedade sobre os gastos em saúde foram, nos países centrais, condições objetivas para o avanço da a valiação dos serviços de saúde como forma de racionalização dos gastos. No Brasil isto não se deu, em parte, devido ao pouco desenvolvimento do seguro privado e também devido à "socialização" dos custos, com repasse de recursos públicos à iniciativa privada, sem o correspondente desenvolvimento de mecanismos de controle pela sociedade.

3) As avaliaçōes de cunho tecnológico, nos países centrais, estão relacionadas à existência de um parque industrial desenvolvido e muito competitivo. No Brasil, o parque industrial nesta área é pouco desenvolvido, o que toma o país um consumidor indiscriminado destas tecnologias, sem a necessária avaliação.

4) Finalmente, nos países centrais o exercício da cidadania leva a que o pacien te seja respeitado enquanto "consumidor" do serviço de saúde. Este fato influencia na qualidade de tais serviços. No Brasil, a inexistência de mecanismos sociais de controle do Estado e a desigualdade na distribuição da riqueza limitam o papel do paciente como "consumidor".

Quality assessment and evaluation becomes an issue of raising concern as the re-organization of health services in Brazil has been discussed. In this review, we search for the historical background of health services evaluation and present the contemporary proposals for a systematic quality evaluation. The 
analyses of the studies reviewed show two major methodological trends in this area: the Rationalizing-Corporative and the Antropological-Social Studies. Most of both types of studies derive from the work on health services evaluation developed during the last twenty years by Avedis Donabedian.

\section{REFERÊNCIAS BIBLIOGRÁFIC IS}

1. ADAY, Lu Ann; ANDERSEN, Ronald $M$. The National Profile of Acess to Medical Care: Where do We Sand? Am. J. Public Health 74(12): 133 1-39, 1984.

2. ADAY, Lu Ann; ANDERSEN, R. A Framework for the S udy of Acess to Medical Care. Health Serv. Res. 9(3): 208-20, 1974.

3. AROUCA, A. S. S. "O Dilema Preventivista. Contribuiçáo para a Compreensão e Crf́tica da Medicina Preventiva". Tese de doutora do apresentada à Faculdade de Ciências Médicas da UNICAMP. mimeo. Campinas, 1975.

4. Bs ',OK, R. H.; LOHR, K. Eff icacy, Effectiveness, Variations and Quality: Boundary-Crossing Research. Med. Care 23(5): 710-22, 1985.

5. BUSSO, Nelida Fernandez. Perspectivas de la Auditoria Médica. Medicina y Sociedad 5(3): 90-97, 1982.

6. CCCIRANE, A. L. Friectiveness and Efficiency Random Reflection on the Health Services. Nuffield Provincial Hospitals Trust, London, 1972.

7. DONABEDIAN, Avedis; WHEELER, Hohn R. C.; WYSZEWIANSKI, Leon. Quality, Cost, and Heahit: An Integrative Model. Med. Care 20(10): 1975-92, 1982.

8. DONABEDIAN, A. Evaluating the Quality of Medical Care. Milbank Mem. Fund. Q. 44:166, Part 2, 1966. - The Quality of Medical Care: A Concept in Search of a Definition. J. Fam. Practic. 3(9): 277-284, 1979. - Promoting Quality Through Evaluating the Process of Patient Care. Med. Care 6(3): 181-202, 1968.

9. DONABEDIAN, A. The Quality of Medical Care. Science $200,1978$.

10. DONNANGELO, M. C. "O Médico e o Mercado de Trabalho". Tese de doutorado apresentada ao DMPS/USP. mimeo. São Paulo, 1972.

11. DRUMMOND, M. F.; STODDART, Greg L.; TORRANCE, George W. Methods for the Economics Evaluation of Health Care Programmes. Oxford Medical Publicetions, 1988.

12. FELDSTEIN, Paul J. The Emergence of Market Competition in the U. S. Health Care System. Its Causes, Likely Structure, and Implications. Health Policy 6(1-20), 1986.

13. FLEXNER, A. Medical Education in United States and Canada: Report to Carnegie Foundation for Advistement of Teaching. Merrymount Press, New York, 1)!0.

14. FUCHS, Victor R. The "Rationing" of Mt. Iical Care. $N$. Engl. J. Med. 311(24): 572-5.73, 1984 
15. GARRO, Linda Young; YOUNG, James C. Atención de $S$ alud en $M$ inorias Etnicas Rurales. Algunas Observaciones Antropológicas. Bio. Of. Sanit. Panam. 95(4): 333-44, 1983.

16. GONZA LES, L. La Calidad de la Atención Médica: Cristus de Los Métodos Evalutivos. Acta Med. Col. 9(2): 60-4, 1984.

17. HI LL, A. B. the Clinical Trial, N. Eng. J. Med. 247:113, 1952.

18. HULKA, Barbosa S.; KUPPER, Lawrence L; CASSEL, John C. Determinants of Physician Utilization: Approach to a Service - Oriented Classification of Symptons. Med. Care 104: 300-309, 1972.

19. KLARMAN, H. Application of Cost-Benefit Analysis to the Health Services and the Special Case of Technologic Innova. tion. Int. J. Health Serv., 4(4): 325, 1974.

Application of Cost-Benefit Analysis to Health Systems Technology. J. Occup. Med., 16:172, 1974.

20. LEBOW, Jay L. Consumer Assessments of the Quality of Medical Care. Med. Care 12(4): 328-37, 1974.

21. LEMBCKE, Paul A. Evolution of Medical Audit. JAMA 199 (8) $111-18,1967$.

22. NOGUEIRA, R. P. "Medicina Interna e Cirúrgica: a Formaçāo Social da Prática Médica". Tese de mestrado apresentada ao Instituto de Medicina Social/UFRJ. mimeo. Rio de Janeiro, 1977.

23. Oficina de Evaluación Tersológica (OPAS); Evaluación de la Eficacia y Securidad de la Tecnología Médica: Estudo de Casos. cap. 3, 1978.

24. PEREZ ARIAS, Elsa B.; FELLER, Jorge J, El Control de los Sistemas de Atención Médica Conctpisualización y Necánica Operativa. Mcdicina y Sociedade $6(6): 239 \cdot 246$, 1983.

25. PORTERFIELD, John D. Evaluatiòn of Patients: Cuinuan Revisited. Bull. N. Y. Acad. Med. 52 (1): 30-8, 1976.

26. SOMMERS, Paul A. Participación Activa de los Consumidores en el Sist. de Prestación de Serv. de Salud: Una Evaluación de la Satisf. Paciente Bol. Of. Sanit. Panam. 94 (1): 54-75, 1983.

27. SONIS, Abraam Y cols. Atención de la Salud. Parte II, cap. 4, Editorial "E1 Ateneo". Buenos Aires, 1984.

28. WARE JR., John E. Effects of Acquiescent Response Set on Patient Satisfaction Rating. Med. Care 16 (4): 327-36, 1978.

SNYDER Mary K.; CHY, Godwin C. Consumer Perceptions of Health Care Services: Implication for Academic Medicine. J. Med. Educ. 50: 839-48, 1975.

- Scales for Measuring General Health Percentions. Health Seri. Res. Winter: $396,415,1976$.

29. WARNER, Kenneth E.; HUTTON, Rebeca C. Cost-Benefit and Cost-Effectiveness Analysis in Health Care. Med. Care 18 (11): 1069-84, 1980.

30. WEISNTEIN, M.; STASON, W. Foundations of Cust-Effectiveness Analysis for Health and Medical Practices. $N$. Engl. j. Med., 296:716, 1977.

_ - ; _ - Allocation of Resources to Manage Hypertension. N. Engl. J. Med., 296:73, 1977. 